\title{
Age-dependent effect of PPAR $\gamma$ agonist pioglitazone on kidney signaling in borderline hypertensive rats
}

\author{
Linda Grešová ${ }^{1}$, Miroslava Kvandová1,2, Peter Kvasnička ${ }^{3}$ and Ima Dovinová ${ }^{1}$ \\ ${ }^{1}$ Institute of Normal and Pathological Physiology, Centre of Experimental Medicine, Slovak Academy of Sciences, Bratislava, \\ Slovakia \\ ${ }^{2}$ Center for Cardiology, Cardiology I - Laboratory of Molecular Cardiology, University Medical Center of the Johannes \\ Gutenberg-University Mainz, Mainz, Germany \\ ${ }^{3}$ Institute of Particle and Nuclear Physics, Faculty of Mathematics and Physics, Charles University, Prague, Czech Republic
}

\begin{abstract}
The peroxisome proliferator-activated receptor $\gamma(\operatorname{PPAR} \gamma)$ is a nuclear receptor and nutrition factor which takes part in the cellular signaling by several agonists such as pioglitazone. PPAR $\gamma$ can serve as potential target in treatments of metabolic syndrome diseases and/or hypertension. In the present study we investigated the effects of pioglitazone, a PPAR $\gamma$ agonist, on hypertension development in young and adult borderline hypertensive rats (BHR). In renal signaling we observed connections between PPAR $\gamma$ and Nrf2, antioxidant in adult animals and differences between young and adult BHR in Nrf2-activated detoxificant outputs (NQO1, HO-1) and NO-synthases. Blood pressure in animals had been detected by cuff plethysmography, cell signaling in the kidney was studied by gene expression determination using qPCR, and nitric oxide synthase (NOS) activity was measured by radioactive detection. Pioglitazone treatment in adult BHR caused no detectable changes in antioxidant and detoxificant responses. The main effects were observed in blood pressure improvement, endothelial NOS expression and NOS activities in both young and adult BHR.
\end{abstract}

Key words: Age-dependence - BHR - Kidney - PPAR $\gamma-$ Nrf2 - NOS - Hypertension

\section{Introduction}

The peroxisome proliferator-activated receptor $\gamma(\operatorname{PPAR} \gamma)$ is a nuclear receptor and nutrition factor in hypertension (Kvandová et al. 2016; Tain et al. 2016). The activity of PPAR $\gamma$ is influenced by a variety of extracellular ligands - glitazones (rosiglitazone, pioglitazone and troglitazone) and intracellular ligands - prostaglandins, leukotrienes, and a-lipoic acid (Houseknecht et al. 2002).

PPAR $\gamma$ activation takes part in the regulation of lipid metabolism, glucose homeostasis and anti-inflammatory response as well as in several cell signaling pathways (Polvani et al. 2012). PPARs undergo a conformational change that causes the translocation to the nucleus and the heterodimerization with another nuclear receptor, the retinoid $\mathrm{X}$ receptor

Correspondence to: Ima Dovinová, Institute of Normal and Pathological Physiology, Centre of Experimental Medicine, Slovak Academy of Sciences, Sienkiewiczova 1, 81371 Bratislava, Slovakia E-mail: ima.dovinova@savba.sk
(RXR) (Mello et al. 2009). The PPAR-RXR heterodimer then binds a DNA portion in the promoter region of target genes, called peroxisome proliferator response element (PPRE), modulating the expression of several genes involved in different physiological or pathological processes (Kliewer et al. 1992). PPAR $\gamma$ and Nrf2-ARE sytem are connected by a positive feedback. PPAR $\gamma$ may also regulate and modulate other systems, such as the renin-angiotensin system (RAS) and NO-synthases (Kvandova et al. 2016, 2017). PPAR $\gamma$ activation may improve dysregulation in metabolic syndrome and hypertension (Chan et al. 2010; Dovinová et al. 2013).

Blood pressure (BP) regulation is an important and complex process. There are several mechanisms that coordinate BP outputs. Nrf2, which is a redox-sensitive nuclear transcription factor exposed to oxidative stress or noxious attacks. It is involved in the induction of antioxidative, detoxificant, and other cytoprotective genes, which provide protection against oxidative stress-induced damage in a variety of cardiovascular diseases and in hypertension (Majzunova et al. 2013; Barančík et al. 2016). The positive 
feedback loop between PPAR $y$ and Nrf2 sustains the expression of both transcription factors and their antioxidant genes. Activation of Nrf2 and PPAR $\gamma$ pathways has ameliorating effects on metabolic disorders and injuries caused by oxidative stress (Lee 2017; Kvandova et al. 2018). After activation, Nrf2 enters nucleus, binds to the anti-oxidant response element (ARE) and produces $\mathrm{NAD}(\mathrm{P}) \mathrm{H}$ :quinone oxidoreductase-1 (NQO1), heme oxygenase-1 (HO-1), glutathione S-transferase, as well internal antioxidants of different superoxide dismutase (SOD) isoforms ( $\mathrm{Li}$ et al. 2008; Lob et al. 2010).

In $\mathrm{BP}$ regulation and generally in the cardiovascular system, NO bioavailability plays a protective role in several tissues (vascular tissues, brain stem, kidney). Oral intake of pioglitazone (PPAR $\gamma$ agonist) significantly abrogated adverse molecular events in metabolic syndrome rats and increased NO level in rostral-ventrolateral medulla of brain stem $(\mathrm{Wu}$ et al. 2014). PPAR $\gamma$ can modulate several signaling pathways: it affects the insulin signaling pathway by modulation of expression and/or phosohorylation of signaling molecules through the PI3/Akt/eNOS pathways (Kvandová et al. 2017). Deficiency of endothelial NO-synthase (eNOS) due to endothelial cell dysfunction plays an important role in the pathophysiology of cardiovascular diseases (hypertension, atherosclerosis) as well as in renal injuries (Nakagawa et al. 2011). Aging is a universal process that affects all organs. Agerelated disruptions in cellular homoeostasis result in decline of organ functions and responsiveness to physiological stress. A gradual decline in renal function occurs in most healthy individuals as they age (Coresh et al. 2003), and the amount of glomerular, vascular and interstitial scarring in the renal tissue of healthy adults increases with age (Rule et al. 2010).

In the present study we investigated the effects of pioglitazone in the hypertension model of young and adult borderline hypertensive rats (BHR). Our study was focused on changes in BP and on renal signaling in young and adult BHR animals after treatment with PPAR $\gamma$ agonist pioglitazone. We studied the effects of pioglitazone on BP development and on the components of the antioxidant SOD1-3 and detoxificant NQO1 and HO-1 responses, and on the NOS signaling pathway.

\section{Materials and Methods}

Experimental design - animal model and treatment protocol

In our study young (5-week old) and adult (12-week old) male borderline hypertensive rats (BHR; offspring of spontaneously hypertensive dams and Wistar-Kyoto sires) were used. The rats were allowed one week to acclimatize to the lab conditions, and were housed three per cage: temperature $22-24^{\circ} \mathrm{C}$, humidity $45-60 \%$, a $12 / 12 \mathrm{~h}$ light/ dark cycle. Young and adult BHR animals were divided into two groups: control group (treated with saline solution) and PIO group (treated with pioglitazone by oral gavage, $10 \mathrm{mg} / \mathrm{kg} /$ day during 10 days). All animal experiments were approved by the Department of Animal Wellness, State Veterinary and Food Administration of the Slovak Republic and carried out in accordance with the Guidelines of the Animal Research and Care Committee of the Institute of Normal and Pathological Physiology of the Slovak Academy of Sciences.

\section{Blood pressure determination}

Systolic BP was measured non-invasively by tail cuff plethysmography using a Statham Pressure Transducer P23XL (Hugo Sachs, Germany). All groups of rats (control group and PIO group of young and adult BHR) have been measured between 08:00 a.m. and 11:00 a.m. as described in detail previously (Puzserova et al. 2013). BP measurements were performed before, during (every three days), and after the treatment period. Each value was calculated as mean of seven measurements.

\section{Gene expression determination}

Expression levels of selected genes were determined by realtime quantitative polymerase chain reaction (RT-qPCR). Total RNA from the renal cortex samples was isolated with TRIsure reagent (Bioline) according to manufacturer's protocol. The isolated total RNA was quantified spectrophotometrically at $260 \mathrm{~nm}$ using a Nanodrop 2000 UV-VIS (Thermo Scientific, USA). The purity of RNA was measured at 260/280 $\mathrm{nm}$ (rate $\sim 2.0$ ) and 260/230 (rate range: 1.8-2.2) for elimination of protein, phenol or other contaminants.

\section{cDNA preparation}

Reverse transcription reaction was performed using a SensiFAST $^{\mathrm{TM}}$ cDNA Synthesis Kit (Bioline, UK) on the Mastercycler Personal (Eppendorf, Germany) according to manufacturer's protocol. Real-time polymerase chain reaction by amplification of cDNA was performed on a CFX96 Real-Time PCR detection system (Bio-Rad, USA) using the Sensi FAST SYBR No ROX kit (Bioline, UK). PCR reaction for each sample was carried out in duplicate for all cDNA. Samples were measured using the Bio-Rad CFX Manager Software (version 2.0). Specific primer pairs were used to amplify the genes studied (PPAR $\gamma$, Nrf2, HO-1, NQO1, SOD1, SOD2, SOD3 and eNOS). $\beta$-actin was used as the "housekeeping" gene (Dovinová et al. 2013, Kvandova et al. 2018). All chemicals used in this study were purchased from Sigma-Aldrich (Germany), Merck Chemicals (Germany); primers were from Metabion $\mathrm{GmbH}$ (Germany). 
Determination of nitric oxide synthase (NOS) activity

Total enzyme activity of NOS was determined in $20 \%$ tissue homogenates of the renal cortex using measurement of the conversion of radioactive $\left[{ }^{3} \mathrm{H}\right]-\mathrm{L}$-arginine (MP Biomedicals, USA) to $\left[{ }^{3} \mathrm{H}\right]$-L-citruline (Bernatova et al. 2002). The product was detected by a Tri-Carb 2910TR liquid scintillator (PerkinElmer, USA) and results were expressed in $\mathrm{pmol} / \mathrm{min} / \mathrm{mg}$ of tissue proteins, determined by the Lowry method.

\section{Statistical analysis}

Data are presented as mean values \pm standard deviation (SD) and the number $(n)$ of independent measurements. BP was analyzed by two-way ANOVA.

Statistical analysis of gene expressions was analysed by one-way ANOVA and unpaired Student's $t$-test and was carried out using an $\mathrm{R}$ language script (R Development Core Team, 2008). We carried out two analyses, one for the effect of pioglitazone on gene expression in adult $\mathrm{BHR}$, the other on the effect of pioglitazone on expression of selected genes in adult and young BHR. A mixed-factor ANOVA model was fitted to each of the two data sets using the R's nlme package (Pinheiro et al. 2018), with contribution of individual animals as random factor. For post-hoc comparisons, we used the glht method of the multcomp R package (Hothorn et al. 2008), with single-step FWER-controlling significance adjustment. Differences were marked as significant for ${ }^{\star} p<0.05$ in all tests.

\section{Results}

\section{Effect of the pioglitazone on blood pressure in BHR}

The effects of treatment with the PPAR $\gamma$ agonist pioglitazone significantly $\left({ }^{*} p<0.05\right)$ decreased the final BP. Changes of final BP between the control and PIO groups were $121.5 \mathrm{mmHg}$ vs. $115 \mathrm{mmHg}$ in young BHR and $148.5 \mathrm{mmHg}$ vs. $136.5 \mathrm{mmHg}$ in adult BHR. The interim BP measurements document a stable BP in young BHR and slightly increasing BP in adult BHR control groups. In the PIO groups, BP monotonically and uniformly decreased during the treatment period in both young and adult rats.

Effect of the pioglitazone on Nrf2 outputs in the kidney of BHR

Nrf2 target genes, HO-1 and phase II-detoxifying gene NQO1, were detected in tested BHR after pioglitazone treatment. PPAR $\gamma$ may act directly for Nrf2 activation in kidney, which may be the underlying mechanism of its renoprotective effect (Li et al. 2008). Figure 1 shows that in young
BHR both Nrf2 signaling outputs in the kidney, HO-1 and NQO1 gene expression, were significantly increased, while no such response was observed in adult BHR.

Pioglitazone treatment and administration markedly elevated PPAR $\gamma$ mRNA and Nrf2 mRNA levels and increased gene expression of SOD1, SOD2, and SOD3 compare to control group in young BHR (Kvandová et al. 2018). Table 1 lists PPAR $\gamma$, Nrf2, and SOD isoforms gene expressions, which were not significantly changed in adult BHR.

\section{Effect of the pioglitazone on NO-synthases in the kidney of $B H R$}

In the kidney of young BHR, we observed a significant increase of mRNA eNOS as well as NOS activities. The same effect, with yet higher outputs, was observed in the kidney of adult BHR (see Figure 2).

$$
\text { A }
$$

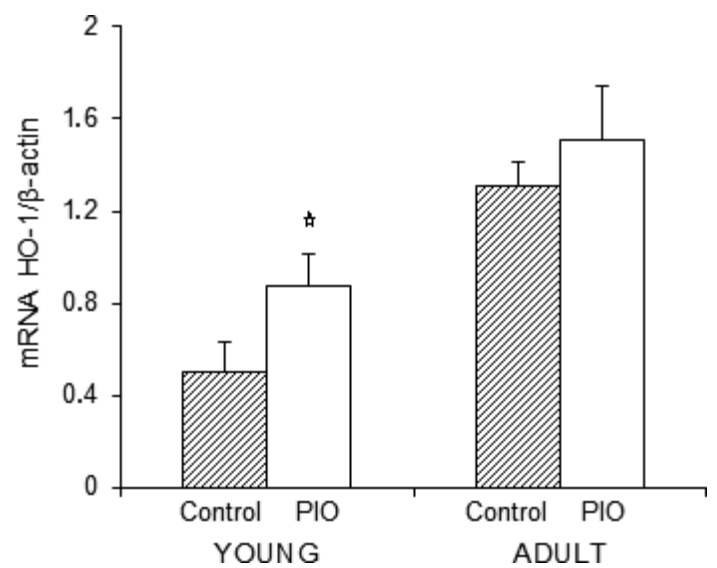

B

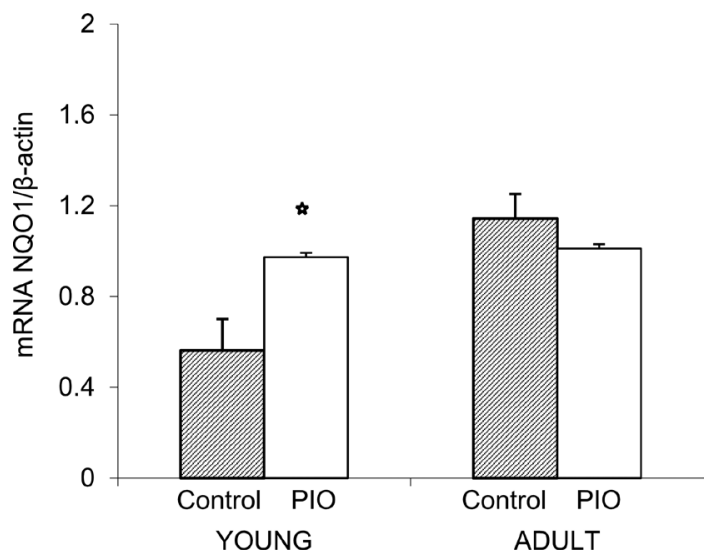

Figure 1. Effect of pioglitazone on gene expression of HO-1 (A) and NQO1 (B) in the kidney of young and adult BHR. Gene expression was normalized on the "housekeeping" gene, $\beta$-actin. Values represent mean $\pm \mathrm{SD},{ }^{*} p<0.05$ versus control group. BHR, borderline hypertensive rats; PIO, rats treated with PPAR $\gamma$ agonist pioglitazone; $n=6$ (for young Control, young PIO group, and adult PIO group), $n=5$ (for adult Control). 
A

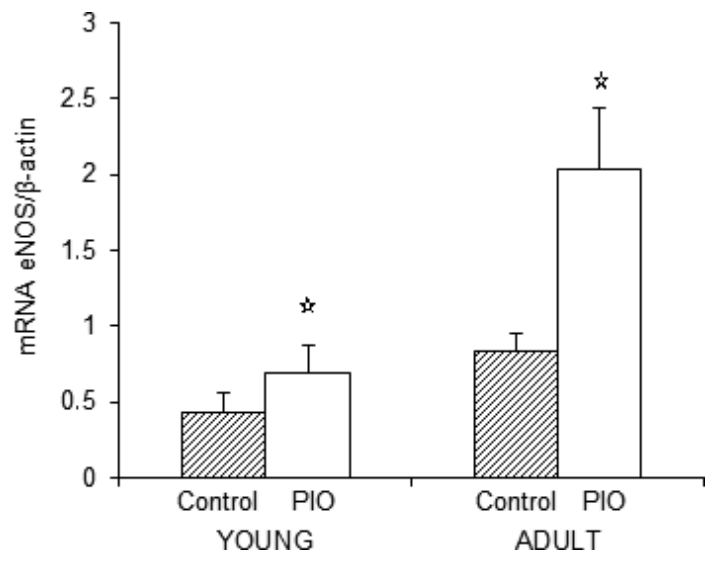

B

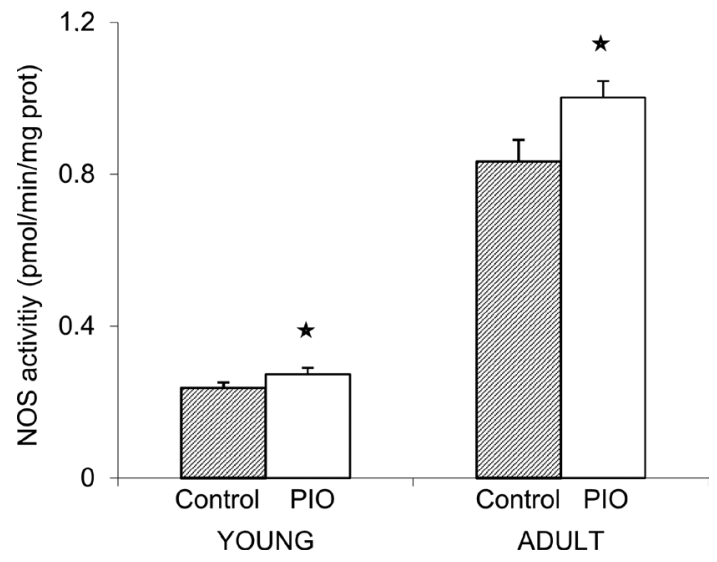

Figure 2. Effect of pioglitazone on gene expression of eNOS (A) and NOS activities (B) in the kidney of young and adult BHR. Gene expression was normalized on the 'housekeeping' gene, $\beta$-actin. Values represent mean $\pm \mathrm{SD},{ }^{*} p<0.05$ versus control group. BHR, borderline hypertensive rats; PIO, rats treated with PPAR $\gamma$ agonist pioglitazone; $n=6$ (for young Control, young PIO group, and adult PIO group), $n=5$ (for adult Control).

\section{Discussion}

Hypertension is an important facet of the metabolic syndrome. Adulthood hypertension and metabolic syndrome can be programmed in response to nutritional insults in early life. PPARs serve as nutrient-sensing signalling, linking nutritional programming to hypertension and the metabolic syndrome. PPAR $\gamma$ are expressed in the kidney and are involved in BP control (Tain et al. 2016). Some studies suggest that administration of a PPAR $\gamma$ agonist (e.g. rosiglitazone) to spontaneously hypertensive rats (SHR) may achieve a BP reduction effect by different mechanisms in different stages of hypertension ( $\mathrm{Li}$ et al. 2010).

In the highly hypertensive rats, SHR, the PPAR $\gamma$ agonist pioglitazone decreases BP only in young animals (Dovinová et al. 2013), but not in adult SHR (Kvandova et al. 2015). In the present study of borderline hypertension in BHR, pioglitazone treatment improved BP. Significant BP decrease was observed in both young and adult BHR.

$\mathrm{BP}$ regulation is an important and complex process including a variety of factors, where normal kidney function plays a considerable role. Experimental and physiological evidence indicates that hypertension development may be affected by the renal PPAR $\gamma$-dependent mechanisms (Tain et al. 2016). In our experiments, we observed no increase in PPAR $\gamma$ and Nrf2 expression in the kidney of adult BHR (Table 1), while pioglitazone administration markedly elevated PPAR $\gamma$ and Nrf2 mRNA levels in young BHR as has been observed previously in young BHR in a similar study (Kvandova et al. 2018). PPAR $\gamma$-dependent activation resulting in SOD1 and SOD2 upregulation and PPAR $\gamma$-dependent activation of Nrf2-mediated elevation of SOD3 expression in young BHR has also been reported by other authors (Zhu et al. 2005; Kvandova et al. 2018). Similar differences between young and adult BHR were also observed in Nrf2 outputs, HO-1 and NQO1 (Figure 1).

In the cardiovascular system, endothelial nitric oxide synthase (eNOS) contributes to the regulation of blood flow and BP and in experimental animals deficiency of eNOS ${ }^{-/-}$ produces hypertension with lower circulating nitrite levels (Wood et al. 2013). eNOS is an essential enzyme to the maintenance of cardiovascular integrity by producing $\mathrm{NO}$, which is a key molecule with multiple functions, including vasodilation. Therefore, dysregulation of eNOS is thought to contribute to pathogeneses of certain vascular diseases, such as atherosclerosis and hypertension. eNOS is regulated not only at the level of expression, but also non-genomically, by subcellular targeting, protein-protein interactions, fatty acylation, and phosphorylation. Several specific phosphorylation sites responsible for eNOS activation have been identified. In contrast, there is evidence of phosphorylation

Table 1. Effect of pioglitazone on gene expression of PPAR $\gamma, \mathrm{Nrf2}$, and SOD isoforms in adult BHR

\begin{tabular}{lccccc}
\hline Group & PPAR $y$ & Nrf2 & SOD1 & SOD2 & SOD3 \\
\hline Control $(n=5)$ & $1.74 \pm 0.654$ & $1.49 \pm 0.391$ & $1.16 \pm 0.285$ & $2.30 \pm 0.659$ & $1.23 \pm 0.523$ \\
PIO $(n=6)$ & $1.46 \pm 0.401$ & $1.21 \pm 0.404$ & $1.44 \pm 0.393$ & $1.82 \pm 0.659$ & $1.75 \pm 0.604$ \\
\hline
\end{tabular}

Gene expression was normalized on the "housekeeping" gene, $\beta$-actin. Values represent mean \pm SD. BHR, borderline hypertensive rats; PIO, rats treated with PPAR $\gamma$ agonist pioglitazone. 
sites that decrease eNOS activity. It has been shown that treatment with troglitazone increases NO production by a PPAR $\gamma$-dependent pathway and a PPAR $\gamma$-independent direct effect on eNOS phosphorylation (Cho et al. 2004). Thiazolidinediones (TZD) - PPAR $\gamma$ agonists - are also known to act in part through AMP-activated protein kinase (AMPK) activation. There is growing evidence that AMPK plays a decisive role in normal renal physiology and pathogenesis of hypertension. (Tain and Hsu 2018). In the vasculature, activation of endothelial AMPK has been shown to phosphorylate NOS $^{1177}$, stimulating NOS activities by NO release and subsequent vasodilatation of both large conduit and resistance arteries (Fu et al. 2016). In our experiments on adult BHR we did not observed changes in PPAR $\gamma$ expression, but eNOS expression was increased (Figure 2A). eNOS expression can be activated through PPAR $\gamma$-independent direct effect. It seems that the effect in adult rats was achieved through pioglitazone action. Pioglitazone administration in young and adult BHR improves NOS activities (Figure 2B). Thus, further examination is required to understand the protective effects of TZDs in programmed hypertension and kidney disease, which are exerted via PPAR $\gamma$-independent direct effect or via the PPAR $\gamma$ signaling pathway.

Acknowledgments: The study was supported by grants VEGA 2/0148/17 and APVV-0348-12.

\section{References}

Barančík M, Grešová L, Barteková M, Dovinová I (2016): Nrf2 as a key player of redox regulation in cardiovascular diseases. Physiol Res. (Suppl. 1) 65, S1-S10

Bernatova I, Pechanova O, Babal P, Kysela S, Stvrtina S, Andriantsitohaina R (2002): Wine polyphenols improve cardiovascular remodeling and vascular function in NO-deficient hypertension. Am. J. Physiol. Heart Circ. Physiol. 282, H942-948 https://doi.org/10.1152/ajpheart.00724.2001

Chan SH, Wu KL, Kung PS, Chan JY (2010): Oral intake of rosiglitazone promotes a central antihypertensive effect via upregulation of peroxisome proliferator-activated receptor-gamma and alleviation of oxidative stress in rostral ventrolateral medulla of spontaneously hypertensive rats. Hypertension 55, 1444-1453 https://doi.org/10.1161/HYPERTENSIONAHA.109.149146

Cho DH, Choi YJ, Jo SA, Jo I (2004): Nitric oxide production and regulation of endothelial nitric-oxide synthase phosphorylation by prolonged treatment with troglitazone: evidence for involvement of peroxisome proliferator-activated receptor (PPAR) gamma-dependent and PPARgamma-independent signalling pathways. J. Biol. Chem. 279, 2499-2506 https://doi.org/10.1074/jbc.M309451200

Coresh J, Astor BC, Greene T, Eknoyan G, Levey AS (2003): Prevalence of chronic kidney disease and decreased kidney function in the adult US population: Third National Health and Nutrition Examination Survey. Am. J. Kidney Dis. 41, 1-12 https://doi.org/10.1053/ajkd.2003.50007
Dovinová I, Barancik M, Majzunova M, Zorad S, Gajdosechová L, Gresová L, Cacanyiova S, Kristek F, Balis P, Chan JY (2013): Effects of PPAR $\gamma$ agonist pioglitazone on redox-sensitive cellular signalling in young spontaneously hypertensive rats. PPAR Res. 2013, 541871 https://doi.org/10.1155/2013/541871

Fu J, Han Y, Wang J, Liu Y, Zheng S, Zhou L, Jose PA, Zeng C (2016): Irisin lowers blood pressure by improvement of endothelial dysfunction via AMPK-Akt-eNOS-NO pathway in the spontaneously hypertensive rat. J. Am. Heart Assoc. 5, e003433 https://doi.org/10.1161/JAHA.116.003433

Hothorn T, Bretz F, Westfall P (2008): Simultaneous inference in general parametric models. Biom. J. 50, 346-363 https://doi.org/10.1002/bimj.200810425

Houseknecht KL, Cole BM, Steele PJ (2002): Peroxisome proliferator-activated receptor gamma (PPAR $\gamma)$ and its ligands: a review. Domest. Anim. Endocrinol. 22, 1-23 https://doi.org/10.1016/S0739-7240(01)00117-5

Kliewer SA, Umesono K, Noonan DJ, Heyman RA, Evans RM (1992): Convergence of 9-cis retinoic acid and peroxisome proliferator signalling pathways through heterodimer formation of their receptors. Nature 358, 771-774 https://doi.org/10.1038/358771a0

Kvandova M, Barancik M, Balis P, Puzserova A, Majzunova M, Dovinova I (2018): The peroxisome proliferator-activated receptor gamma agonist pioglitazone improves nitric oxide availability, renin-angiotensin system and aberrant redox regulation in the kidney of pre-hypertensive rats. J. Physiol. Pharmacol. 69, 231-243

Kvandová M, Dovinová I (2017): Functioning of the PPAR $\gamma$ and its effect on cardiovascular and metabolic diseases. In: Metabolic Syndrome. SMgroup, pp. 1-41, Dover, USA

Kvandova M, Kratka D, Balis P, Barancik M, Dovinova I (2015): The effect of PPARg agonist - pioglitazone on hypertension and redox regulation in young and adult SHR. Cardiol. Letters 24, 240-243

Kvandová M, Majzúnová M, Dovinová I (2016): The role of PPARgamma in cardiovascular diseases. Physiol. Res. (Suppl. 3) 65, S343-S363

Lee C (2017): Collaborative power of Nrf2 and PPAR $\gamma$ activators against metabolic and drug-induced oxidative injury. Oxid. Med. Cell Longev. 2017, 1378175 https://doi.org/10.1155/2017/1378175

Li R, Zhang H, Wang W, Wang X, Huang Y, Huang C, Gao F (2010): Vascular insulin resistance in prehypertensive rats: role of PI3kinase/Akt/eNOS signalling. Eur. J. Pharmacol. 628, 140-147 https://doi.org/10.1016/j.ejphar.2009.11.038

Li W, Khor TO, Xu C, Shen G, Jeong WS, Yu S, Kong AN (2008): Activation of Nrf2-antioxidant signalling attenuates NFkappaBinflammatory response and elicits apoptosis. Biochem. Pharmacol. 76, 1485-1489 https://doi.org/10.1016/j.bcp.2008.07.017

Lob HE, Marvar PJ, Guzik TJ, Sharma S, McCann LA, Weyand C, Gordon FJ, Harrison DG (2010): Induction of hypertension and peripheral inflammation by reduction of extracellular superoxide dismutase in the central nervous system. Hypertension 55, 277-283 https://doi.org/10.1161/HYPERTENSIONAHA.109.142646 
Majzunova M, Dovinova I, Barancik M, Chan JY (2013): Redox signalling in pathophysiology of hypertension. J. Biomed. Sci. 20, 69 https://doi.org/10.1186/1423-0127-20-69

Mello T, Polvani S, Galli A (2009): Peroxisome proliferator-activated receptor and retinoic $\mathrm{X}$ receptor in alcoholic liver disease. PPAR Res. 2009, 748174 https://doi.org/10.1155/2009/748174

Nakagawa T, Tanabe K, Croker BP, Johnson RJ, Grant MB, Kosugi T, Li Q (2011): Endothelial dysfunction as a potential contributor in diabetic nephropathy. Nat. Rev. Nephrol. 7, 36-44 https://doi.org/10.1038/nrneph.2010.152

Pinheiro J, Bates D, DebRoy S, Sarkar D, R Core Team (2018): Nlme: Linear and Nonlinear Mixed Effects Models. R package version 3.1-137

Polvani S, Tarocchi M, Galli A (2012): PPAR and oxidative stress: Con $(\beta)$ catenating NRF2 and FOXO. PPAR Res. 2012, 641087 https://doi.org/10.1155/2012/641087

Puzserova A, Slezak P, Balis P, Bernatova I (2013): Long-term social stress induces nitric oxide-independent endothelial dysfunction in normotensive rats. Stress 16, 331-339 https://doi.org/10.3109/10253890.2012.725116

R Development Core Team (2008): R: A language and environment for statistical computing. R Foundation for Statistical Computing, Vienna, Austria.

Rule AD, Amer H, Cornell LD, Taler SJ, Cosio FG, Kremers WK, Textor SC, Stegall MD (2010): The association between age and nephrosclerosis on renal biopsy among healthy adults. Ann. Intern. Med. 152, 561-567 https://doi.org/10.7326/0003-4819-152-9-201005040-00006

Tain YL, Hsu CN (2018): AMP-activated protein kinase as a reprogramming strategy for hypertension and kidney disease of developmental origin. Int. J. Mol. Sci. 19, E1744 https://doi.org/10.3390/ijms19061744

Tain YL, Hsu CN, Chan JY (2016): PPARs link early life nutritional insults to later programmed hypertension and metabolic syndrome. Int. J. Mol. Sci. 17, 20 https://doi.org/10.3390/ijms17010020

Wood KC, Cortese-Krott MM, Kovacic JC, Noguchi A, Liu VB,Wang X, Raghavachari N, Boehm M, Kato GJ, Malte Kelm M, Gladwin MT (2013): Circulating blood eNOS contributes to the regulation of systemic blood pressure and nitrite homeostasis. Arterioscler. Thromb. Vasc. Biol. 33, 10.1161 https://doi.org/10.1161/ATVBAHA.112.301068

Wu KL, Chao YM, Tsay SJ, Chen CH, Chan SH, Dovinova I, Chan JY (2014): Role of nitric oxide synthase uncoupling at rostral ventrolateral medulla in redox-sensitive hypertension associated with metabolic syndrome. Hypertension 64, 815-824 https://doi.org/10.1161/HYPERTENSIONAHA.114.03777

Zhu H, Itoh K, Yamamoto M, Zweier JL, Li Y (2005): Role of Nrf2 signaling in regulation of antioxidants and phase 2 enzymes in cardiac fibroblasts: protection against reactive oxygen and nitrogen species-induced cell injury. FEBS Lett. 579, 3029-3036 https://doi.org/10.1016/j.febslet.2005.04.058

Received: January 7, 2019

Final version accepted: January 22, 2019 